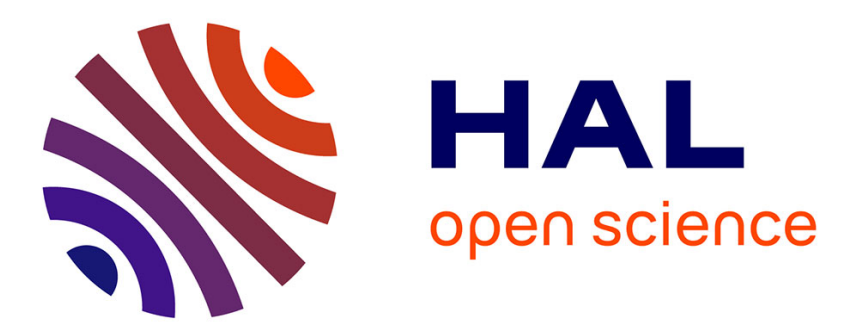

\title{
Positronium reemission yield from mesostructured silica films
}

\author{
Liszkay Laszlo, Catherine Corbel, Patrice Perez, Pierre Desgardin, M.-F.
}

Barthe, Toshiyuki Ohdaira, Ryoichi Suzuki, Paolo Crivelli, Ulisse Gendotti, André Rubbia, et al.

\section{To cite this version:}

Liszkay Laszlo, Catherine Corbel, Patrice Perez, Pierre Desgardin, M.-F. Barthe, et al.. Positronium reemission yield from mesostructured silica films. 2007. hal-00201089v3

\section{HAL Id: hal-00201089 \\ https://hal.science/hal-00201089v3}

Preprint submitted on 29 Jan 2008

HAL is a multi-disciplinary open access archive for the deposit and dissemination of scientific research documents, whether they are published or not. The documents may come from teaching and research institutions in France or abroad, or from public or private research centers.
L'archive ouverte pluridisciplinaire HAL, est destinée au dépôt et à la diffusion de documents scientifiques de niveau recherche, publiés ou non, émanant des établissements d'enseignement et de recherche français ou étrangers, des laboratoires publics ou privés. 


\section{Positronium re-emission yield from mesostructured silica films}

L. Liszkay ${ }^{*}$, C. Corbel, P Perez

DSM/IRFU \& IRAMIS, CEA Saclay F-91191 Gif-sur-Yvette Cedex, France

P. Desgardin, M.-F. Barthe

CNRS-CERI, $3 A$ rue de la Férollerie, F-45071 Orléans Cedex 2, France

T. Ohdaira, R. Suzuki

AIST, Tsukuba, Ibaraki 305-8568, Japan

P. Crivelli, U. Gendotti, A. Rubbia

Institut für Teilchenphysik, ETHZ, CH-8093 Zürich, Switzerland

M. Etienne, A. Walcarius

LCPME, CNRS-Nancy-Université, 405 rue de Vandoeuvre, F-54600 Villers-lès-Nancy, France

The re-emission yield of ortho-positronium (o-Ps) into vacuum outside mesoporous silica films on glass is measured in reflection mode with a specially designed lifetime (LT) spectrometer. Values as high as $40 \%$ are found. The intensity of the $142 \mathrm{~ns}$ vacuum LT is recorded as a function of re-emission depth. The LT depth profiling is correlated to the $2 \gamma$ and $3 \gamma$ energy ones to determine the annihilation characteristics inside the films. Positron lifetime in capped films is used to determine the pore size. For the first time, a set of consistent fingerprints for positronium annihilation, $o$-Ps re-emission into vacuum, and pore size, is directly determined in surfactant-templated mesoporous silica films.

(Accepted for publication in Applied Physics Letters)

Characterization of the pore organization, size and volume in mesostructured silica films is one key step for controlling their properties - such as dielectric constant, refractive index, permeability, absorption, etc. in various applications ${ }^{1,2}$. Positron $\left(\mathrm{e}^{+}\right)$annihilation has been used to investigate more specifically the pore volume distribution vs. depth using low energy $\mathrm{e}^{+}$beams ${ }^{3}$. However, the $\mathrm{e}^{+}$lifetime (LT) spectra obtained by using conventional LT spectrometers have been difficult to interpret due to the presence of long lifetimes in the range of 40-142 ns. It has been early recognized that lifetimes of $142 \mathrm{~ns}$ indicate that positronium (Ps) atoms, i.e a positron-electron $\left(\mathrm{e}^{+}-\mathrm{e}^{-}\right)$bound pair, can annihilate from triplet spin orthopositronium (o-Ps) vacuum-like states present either in the films or after escape from the film surface, in vacuum.

To determine whether the $142 \mathrm{~ns}$ vacuum LT exists inside the films, several authors have compared the LT spectra in the films before and after capping with thin layers $(20-50 \mathrm{~nm})^{3,4}$. The vacuum LT was found to disappear and only shorter lifetimes, 40-50 ns, with high intensity, $>10 \%$, were detected after capping. It follows that $o$-Ps quenching takes place in mesoporous films as in other insulating materials. However, the quenching is much less efficient in the films than in polymers or molecular materials where the quenched lifetimes are much shorter $(<3 \mathrm{~ns})$. The presence of $o$-Ps in the films has recently been used to successfully convert a high intensity $\mathrm{e}^{+}$beam pulse into $o$-Ps gas confined in the mesopores with decay times and densities sufficient to observe Ps-Ps interaction ${ }^{5}$. A step further would be to initiate a novel physics by achieving Bose-Einstein condensation of positronium ${ }^{6}$.

\footnotetext{
* electronic mail: lliszkay@mail.kfki.hu; on leave from: KFKI Research Institute for Nuclear and Particle Physics, H-1525 Budapest, P. O. Box 49, Hungary.
} 
Concurrent with the above studies, other investigators have focussed their interest on Ps escape into vacuum. To demonstrate that mesoporous silica films reemit Ps, the AIST time of flight (TOF) spectrometer was used to record the $o$-Ps energy distribution in vacuum versus the $\mathrm{e}^{+}$beam energy ${ }^{7}\left(E_{e+}\right)$. This equipment gives evidence that Ps re-emission (PsR) takes place not only for $\mathrm{e}^{+}$implanted near the surface $\left(E_{e_{+}}<0.5 \mathrm{keV}\right)$, but also deeper in the films. As the implanted depth increases from 0 to $300 \mathrm{~nm}$, a decrease is observed in the kinetic energy at which Ps is reemitted. This property singles out mesostructured silica films as potential structures to prepare clouds of low energy $o$-Ps $(<0.1 \mathrm{eV})$ in vacuum. Attention has recently been attracted to the novel experiments in fundamental physics ${ }^{8}$ that can be achieved once a low energy $o$-Ps cloud with high density is available. Its interaction with an antiproton beam provides a method for the direct or indirect production of neutral antihydrogen atoms, suitable for direct gravity measurements on antimatter ${ }^{8}$.

With regard to the production of Ps clouds in vacuum, review of the literature shows that appropriate equipments for performing direct measurements of the vacuum $o$-Ps re-emission (o-PsR) yield have been unavailable. This Letter reports such measurements using a novel LT spectrometer constructed at CERN in-line with a $1-6 \mathrm{keV} \mathrm{e} \mathrm{e}^{+}$beam. The vacuum $o$-PsR yield is coupled to a novel fingerprint of Ps annihilation in the films ${ }^{9}$ obtained by recording the annihilation $\gamma$ energy distribution at the CERI $0.5-25 \mathrm{keV} \mathrm{e}^{+}$beam. The two annihilation characteristics that gives the fingerprint for the $2 \gamma$ and $3 \gamma$ Ps annihilation modes are correlated to determine for which $\mathrm{e}^{+}$energy it is suitable to compare the PsR yields between various films. Finally, the pore size distribution in the films is determined by capping the films and recording the $\mathrm{e}^{+} \mathrm{LT}$ spectra with the AIST $\mathrm{e}^{+}$beam tuned to $2 \mathrm{keV}$. The CERN and AIST LT spectra are correlated to determine the size of the pores that reemit $o$-Ps.

The mesoporous CTACl-TEOS films are spin-coated on glass. They are prepared via sol-gel process using tetraethoxysilane (TEOS) as the mineral source for the silica network skeleton precursor and cetyl trimethyl ammonium chloride $(\mathrm{CTACl})$ cationic surfactants as the organic pore generator (porogen) agent. A pure aqueous method is used ${ }^{10}$. The CTACl/TEOS molar ratio, $x$, for the 24 films prepared in Nancy $(\mathrm{NC} x$ ) or Saclay (SC $x$ ) varies over a wide range from 0.07 to 0.22 . The NC0.1 films were prepared with three different thicknesses by $n$ (= 1 to 3 ) consecutive depositions. After deposition, the CTACl-TEOS/Glass samples are treated at $130{ }^{\circ} \mathrm{C}$ and stored in air. They are calcinated at $450{ }^{\circ} \mathrm{C}$ in air immediately before the $\mathrm{e}^{+}$measurements. Patterns, indicating symmetry in pore organization, are absent from the recorded X-ray diffraction patterns.

The $\gamma$ energy distribution in the films (measured at CERI with $0.2-25 \mathrm{keV} \mathrm{e}^{+}$) results from both $2 \gamma$ and $3 \gamma$ annihilation modes. The $2 \gamma$ mode from singlet $\mathrm{e}^{-}-\mathrm{e}^{+}$annihilating pairs (unbound and bound ( $p$-Ps)) contributes to the $511 \mathrm{keV} \pm \Delta \mathrm{E}$ annihilation peak. Its lineshape is defined here by the low momentum fraction, $S_{2 \gamma}$, of $\mathrm{e}^{+}-\mathrm{e}^{-}$pairs annihilating in the energy window: $511 \pm 0.656 \mathrm{keV}$. The $3 \gamma$ mode arises only from $o$-Ps annihilation $(o$-PsA) and contributes to the whole $\gamma$ energy distribution. The $3 \gamma$ yield ${ }^{11}$ directly reflects the $o$-PsA yield, $Y_{3 \gamma}$. The typical features of $S_{2 \gamma}$ and $Y_{3 \gamma}$ versus $E_{e+}$, shown in Fig.1a and b, respectively, for $0.2-25 \mathrm{keV} \mathrm{e}^{+}$implanted in NC0.1/glass, are reproducible. Above 2.4(2) keV, there is a break that develops into a plateau as the number of depositions increases. To discuss the origin of this break, we use Fig.1c where $\mathrm{Y}_{3 \gamma}$ versus $\mathrm{S}_{2 \gamma}$ is plotted with $E_{e+}$ as running parameter. In this plot the breakpoint is well visible even in the thinnest film. Such a plot of two independent annihilation signals provides a direct way for determining the number $i$ of different homogeneous regions and their fingerprint $\left(\mathrm{S}_{i}, Y_{i}\right)$, that contribute to the annihilation signals when the annihilation probability in the regions varies with $E_{e+}$. The linear relationship, $Y_{3 \gamma}\left(S_{2 \gamma}\right)$ in Fig.1c gives direct evidence that, for $E_{e+} \geq 2.4(2) \mathrm{keV}$, positrons annihilate with only two fingerprints, one in glass and the other one in the film. Below the 
breakpoint energy, 2.4(2) keV, the film surface starts to contribute.and the plot deviates from linearity. The slopes in Fig.1c depend on the CTACl/TEOS molar ratio used for the film preparation. The slope is higher in $\mathrm{NC} 0.22$ than in $\mathrm{NC} 0.1$. The lines intersect at $(0.417,0)$ for $E_{e+}=25 \mathrm{keV}$ and define the annihilation fingerprint $\left(S_{g}, Y_{g}\right)$ in glass. As regard $\mathrm{e}^{+}$ annihilation, the film is a homogeneous region where $\mathrm{e}^{+}$annihilate either (1) in the film bulk or (2) at the interface between the film and the glass substrate. The first situation rather than the second one, is consistent with the existence of plateaus for $S_{2 \gamma}$ (Fig.1a) and $Y_{3 \gamma}$ (Fig.1b) when the film thickness increases with the deposition number. It provides evidence that positrons annihilate in the film bulk. The deviation from linearity for $E_{e+} \leq 2.4(2) \mathrm{keV}$ gives evidence that annihilation fingerprints differs in film bulk and surface. The $Y_{3 \gamma}\left(S_{2 \gamma}\right)$ breaking point at 2.4(2) keV gives a fingerprint of annihilation in the film bulk. The comparison of the film fingerprints $(0.487(2), 0.339(1))$ in $\mathrm{NC} 0.22$ and $(0.498(2), 0.135(5))$ in $\mathrm{NC} 0.1$ shows that the $o$-PsA is $\sim 30 \%$ higher $\left(Y_{3 \gamma}\right)$ for $\mathrm{NC} 0.22$ than for NC0.1 and has a lower probability for quenching.

The $Y_{3 \gamma}\left(S_{2 \gamma}\right)$ plot shows that a model with only three annihilation fingerprints $\left(S_{2 \gamma i}, Y_{3 \gamma i}\right)$ in glass, film bulk and surface is sufficient to fit their $\mathrm{e}^{+}$energy dependence. The curves are fitted using VEPFIT ${ }^{12}$ to calculate the probability distribution, $P\left(E_{e+}, z\right)$, as a function of the depth, implantation profile and diffusion lengths in glass and film. In C0.1, the fits can be achieved assuming film thicknesses of 500, 1000 and $1500 \times 10^{-7} \mathrm{~g} \mathrm{~cm}^{-2}$ for the $n=1,2$ and 3 depositions.

To determine whether the high fraction of $o$-PsA for 1-6 keV e ${ }^{+}$(Fig. 2a) has its origin from inside or outside the films, the LT spectra in the films were measured at CERN using a LT beam ${ }^{13}$ based spectrometer equipped with a 200x220 mm wall of four large BGO scintillation $\gamma$ detectors. The wall is parallel to the incoming $\mathrm{e}^{+}$beam and overlooks a space region located from $\sim 55 \mathrm{~mm}$ beyond the sample holder to $\sim 165 \mathrm{~mm}$ ahead of the film. The detection efficiency in reflection mode has been validated for $o$-Ps re-emission energy in the $0.1-1 \mathrm{eV}$ range. Monte-Carlo simulation (using Geant $4^{14}$ ) shows that the detection efficiency for $3 \gamma$ events ${ }^{9}$ is $\sim 2 \%$ lower than that for $2 \gamma$ ones. For NC0.1 and SC0.22, the LT spectra recorded for 1 to $6 \mathrm{keV} \mathrm{e}^{+}$(Fig.2b) can be fitted with a sum of three exponential decay components, $\left(I_{i}, \tau_{i}\right)$, for the annihilation state distribution ${ }^{15}$. The $5 \mathrm{~ns}$ FWHM resolution allows good separation of decays with long lifetimes, $\tau_{i}>\sim 10 \mathrm{~ns}$. Two long LT components, are resolved in NC0.1 whereas only one in SC0.22. The LT values for $1-6 \mathrm{keV} \mathrm{e}^{+}$are independent of the $\mathrm{e}^{+}$ energy. This is not the case for their intensities. For NC0.1, the intensity of the annihilation state with LT, $\tau_{f}=41(1) \mathrm{ns}$, goes through a weak maximum at $3 \mathrm{keV}$. Its value for 1 and 6 $\mathrm{keV}$ differ by $7 \%$. For both type of films, the longest LT decay has the same value, $\tau_{v}=142(5)$ ns. The $o$-Ps re-emission yield into vacuum, $Y_{v}$, in Fig.2b is calculated as the annihilation probability from the $\left(I_{v}, \tau_{v}=142 \mathrm{~ns}\right)$ component. The spectrometer detecting $3 \gamma$ and $2 \gamma$ events with the same efficiency, $Y_{v}$, is thus given by the expression: $Y_{v}=I_{v} \tau_{v} / \sum_{i} I_{i} \tau_{i}$. The values are the highest at $1 \mathrm{keV} \mathrm{e}^{+}: 0.41(1)$ in SC0.22 and 0.18(1) in NC01. Independently of the $\mathrm{e}^{+}$energy, $Y_{v}$ is always higher in $\mathrm{SC} 0.22$ than in $\mathrm{NC} 0.1$. In the range $1-6 \mathrm{keV}$, the yields (Fig. 2b) vary by $\sim 7 \%$ in SC0.22 and NC01.

The AIST LT spectrometer is used to record the $\mathrm{e}^{+}$LT spectra after capping the films with a $20 \mathrm{~nm}$ silica layer. The $270 \mathrm{ps}$ FWHM resolution allows us to resolve three exponential decay components, $\left(I_{i}, \tau_{i}\right)$, for $2 \mathrm{keV} \mathrm{e}{ }^{+}$in capped NC0.22 and SC0.22. For the longer one, the intensity and lifetime values, $\left(I_{c f},(\%), \tau_{c f}(\mathrm{~ns})\right)$ are $(24(1), 44(2))$ in NC0.22 and (36(1), 52(2)) in SC0.22. In models where o-Ps quenching in capped mesoporous films is due to pick-off of electrons at the pore walls, a 44-52 ns LT corresponds to spherical ${ }^{16}$ or cubic $^{17}$ pores of 
$\sim 2.5-3 \mathrm{~nm}$ diameter or $\sim 3-4 \mathrm{~nm}$ length, respectively. This pore size is near the value found with other methods ${ }^{10}$.

The CERI $Y_{3 \gamma}\left(S_{2 \gamma}\right)$ results give the energy value, 2.4(3) $\mathrm{keV}$, where the annihilation characteristics originates from the film bulk in C0.1 and C.022. The vacuum PsR yield from the film bulk correspond to the CERN value $Y_{v}$ at $2.4(3) \mathrm{keV}$. The $Y_{v}$ values are $0.36(2)$ and 0.09(1) for SC0.22 and NC0.1, respectively. The correlation between the LT data before (CERN) and after (AIST) film capping indicates from where in the film the $o$-Ps escapes. The probability for the occupation of $o$-Ps states in the 3-4 nm mesopores is given by the intensity of the AIST LT component $\left(I_{c f}, \tau_{c f}\right)$ determined at $2 \mathrm{keV}$ after film capping. This intensity is quite comparable to the intensity of the 2-2.4 keV CERN LT component $\left(I_{v}, 142\right.$ ns) determined before capping the film and giving the probability for the occupation of $o$-Ps states in vacuum. This correlation provides the first direct evidence that Ps escapes from the mesopores in such films and confirms earlier results for CTAB/TEOS films ${ }^{18}$. Furthermore, using the CERN mean LT values, the $o$-PsA yield in the films can be calculated for $1-6 \mathrm{keV}$ $\mathrm{e}^{+}$. The comparison in Fig. 2a between the calculated CERN values with the experimental $Y_{3 \gamma}$ CERI values is good showing the consistency of the measurements and analysis.

In summary, three types of $\mathrm{e}^{+}$annihilation spectroscopy at CERI, CERN and AIST are coupled to give a complete picture of Ps annihilation and re-emission from CTACl-TEOS films. Using, for the first time, the correlation between $3 \gamma$ and $2 \gamma$ annihilation modes (CERI), it is possible to select the appropriate $\mathrm{e}^{+}$energy range in which to determine the fingerprints of Ps annihilation in the film bulk (CERI), the $o$-Ps re-emission yield (CERN) from the film bulk and the pore size distribution (AIST) that controls the re-emission. This coupling provides a powerful and reliable method to directly correlate the $o$-Ps re-emission to the pore volume distribution in the film bulk and, consequently, to optimize the re-emission property as a function of the film engineering. 


\section{References}

1. D. Grosso D, F. Cagnol, G. J. de A. A. Soler-Illia, E. L. Crepaldi, H. Amenitsch, A. Brunet-Bruneau, A. Bourgeois and C. Sanchez, Adv. Funct. Materials 14, 309 (2004).

2. M. Etienne, A. Quach, D. Grosso, L. Nicole, C. Sanchez, A. Walcarius, Chem. of Materials 19, 844 (2007).

3. D.W. Gidley, H.G. Peng, R.S. Vallery, Annual Review of Materials Research 36, 49 (2006).

4. C. He, T. Oka, Y. Kobayashi, N. Oshima, T. Ohdaira, A. Kinomura, R. Suzuki, Appl. Phys. Lett. 91, 024102 (2007).

5. D. B. Cassidy, A. P. Mills Jr, Nature 449, 195 (2007).

6. P.M. Platzman, A.P. Mills Jr., Phys. Rev. B 49, 454 (1994).

7. R. S. Yu, T. Ohdaira, R. Suzuki, K. Ito, K. Hirata, K. Sato, Y. Kobayashi, Jun Xu Appl. Phys. Lett. 83, 4966 (2003).

8. P.Perez, A. Rosowsky, Nuclear Instrum. Meth. A 545, 20 (2005).

9. Zs. Kajcsos, L. Liszkay, G. Duplatre, L. Lohonyai, L. Varga, K . Lazar, G. PalBorbely, H.K. Beyer, P. Caullet, J. Patarin, A.P. de Lima, C. Lopes Gil, P.M. Gordo, M.F. Ferreira Marques, Radiation Physics and Chemistry 68, 363-368 (2003).

10. Y. Cohen, K. Landskron, N. Tetretault, S. Fournier-Bidoz, B. Hatton, G. A. Ozin, Adv. Funct. Mater. 15, 593 (2005).

11. M. P. Petkov, M. H. Weber, K. G. Lynn, K. P. Rodbell, Appl. Phys. Lett. 79, 3884 (2001).

12. van Veen, H. Schut, J. de Vries, R.A. Hakvoort and M.R. Ijpma. In: P.J. Schultz, G.R. Massoumi and P.J. Simpson, Editors, Positron Beams for Solids and Surfaces AIP Conf. Proc. Vol. 218, AIP, New York (1990), p. 171.

13. N. Alberola, T. Anthonioz, A. Badertscher, C. Bas, A.S. Belov, P. Crivelli, S.N. Gninenko, N.A. Golubev, M.M. Kirsanov, A. Rubbia, D. Sillou, Nuclear Instr. Meth. A 560, 224 (2006).

14. S. Agostinelliae, J. Allison, K. Amakoe, J. Apostolakisa, H. Araujoaj, P. Arcel, M. Asaig, D. Axeni, Banerjeebi, G. Barrand et al, Nucl. Instr and Meth. A 506, 250 (2003); http://geant4.web.cern.ch/geant4/.

15. Kansy, J., Nucl. Instrum. Methods. A 374, 235 (1996).

16. T. Goworek, K. Ciesielski, B. Jasinska and J. Wawryszczuk, Chem. Phys. Letters 272, 91 (1997).

17. .L. Dull, W.E. Frieze, D.W. Gidley, J. Sun, A.F. Yee, J. Phys. Chem. B 105, 4657 (2001).

18. van Veen, R. Escobar Galindo, H. Schut,W. W. H. Eijt, C. V. Falub, A. R. Balkenende, F. K. de Theije, Mat. Sci. Engineering B 102, 2 (2003). 
Figures

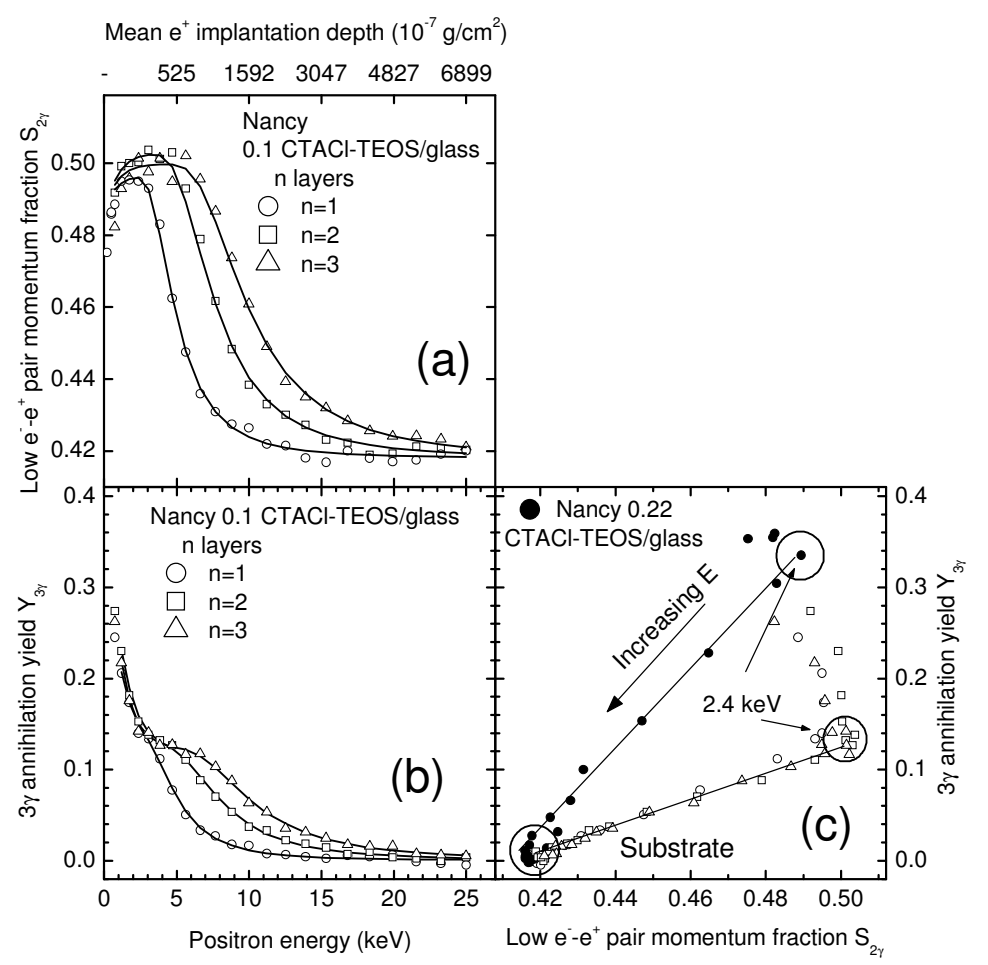

Figure 1

(a) Low e $\mathrm{e}^{-} \mathrm{e}^{+}$pair momentum fraction $S_{2 \gamma}$, (b) $3 \gamma o$-Ps annihilation yield $Y_{3 \gamma}$ vs. $\mathrm{e}^{+}$beam energy and (c) $Y_{3 \gamma}$ vs. $S_{2 \gamma}$ in NC0.10 for 1, 2, 3 coatings and in NC0.22. Three annihilation characteristics for glass, film bulk and surface are used for the fitted lines. 


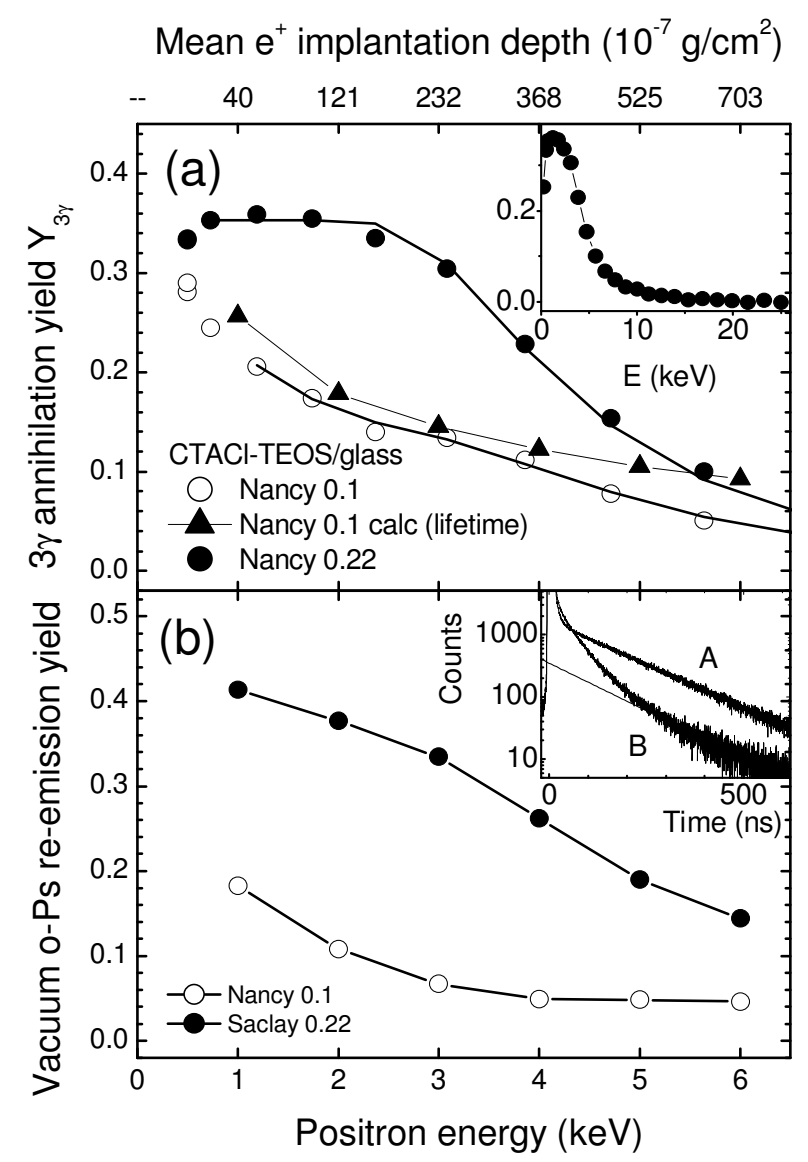

Figure 2

(a) $3 \gamma o$-Ps annihilation yield for 1-6 keV $\mathrm{e}^{+}$recorded at CERI in NC0.10 and NC0.22 or calculated in NC0.1 from CERN vacuum lifetime component (triangles); (b) vacuum $o$-Ps reemission yield in NC0.10 and SC0.22 for $1-6 \mathrm{keV} \mathrm{e}^{+}$and CERN lifetime spectra for $3 \mathrm{keV} \mathrm{e}^{+}$ in the SC0.22 (A) and NC0.1 (B) sample ( insert). 